\title{
Theoretical Study on CO2 Hydrogenation Mediated by Ru-PNP Pincer Complexes: An Implication Towards Rational Catalyst
}

Design

Shahnaz Rohman ${ }^{1}$, Chayanika Kashyap ${ }^{1}$, Amlan Kalita ${ }^{1}$, Sabnam Ullah ${ }^{1}$, Indrani Baruah ${ }^{1}$, Lakhya Mazumder ${ }^{1}$, and Ankur Guha ${ }^{1}$

${ }^{1}$ Cotton University

January 26, 2021

\begin{abstract}
Catalytic CO2 reduction mediated by Ru-PNP pincer complexes has been studied using density functional theory (DFT). Calculations clearly reveal that modification of the PNP pincer framework by introducing planar conjugation in the backbone improves the catalytic efficiency. Activation strain model reveals that reduction of strain in the transition states with modified PNP framework associated with the insertion of $\mathrm{CO} 2$ molecule is responsible for lowering the activation barrier. Calculations also reveal that electron withdrawing substituents at the PNP ligand improves the catalytic performance.
\end{abstract}

\section{Hosted file}

MS-IJQC.pdf available at https://authorea.com/users/297271/articles/505895-theoreticalstudy-on-co2-hydrogenation-mediated-by-ru-pnp-pincer-complexes-an-implication-towardsrational-catalyst-design 